\title{
Adaptation of the maternal heart in pregnancy
}

\author{
Stewart Hunter, Stephen C Robson
}

Major adaptations occur in the maternal cardiovascular system during normal pregnancy and knowledge of these changes is essential to the management of women with cardiovascular disease. Cardiac output increases during pregnancy but the extent and timing of this increase and the underlying causes have been a subject of debate. The controversy was largely the result of differences in investigative techniques and the design of the clinical studies. From the earliest work, of Linhard in $1915,{ }^{1}$ to the present day, investigators have vacillated between the repeatability but inaccuracy of indirect Fick methods and the potential hazards and ethical problems of longitudinal invasive investigations by dye dilution or the direct Fick method. As a result many early studies were cross sectional in design and therefore limited by the wide variations in cardiac output and stroke volume between individuals.

The non-invasive techniques of $M$ mode echocardiography and impedance echocardiography were first used in the 1960 s and 1970s. Cardiac output could be measured serially without risk or discomfort to the subject. Such techniques allowed the design of studies in which each woman acted as her own pre-pregnant control. Determination of cardiac output by impedance cardiography was described by Kubicek et al in $1966 .^{2}$ This technique used changes in transthoracic electrical resistance resulting from ejection of blood into the pulmonary circulation. A comparison with thermodilution measurements during caesarean section ${ }^{3}$ showed a poor correlation with impedance cardiography. de Swiet and Talbert subsequently cast considerable doubt on the assumptions of the Kubicek technique ${ }^{4}$ : the validity of the results obtained with it have subsequently been questioned. On the other hand $M$ mode echocardiography did seem to have some merit for longitudinal pregnancy studies. Stroke volume was calculated by cubing the minor left ventricular internal dimensions. ${ }^{5}$ This method assumed that the left ventricle was an ellipsoid of revolution (rugby ball shaped) so that the long axis was twice the minor axes. When ventricular function and structure are normal this method seems to be valid. However, Fortuin et al showed that dilatation of the left ventricle destroys the essential mathematical relation and with it the validity of the method. ${ }^{6}$ During pregnancy heart volume and end dias- tolic dimension do both increase progressively" ${ }^{78}$ and therefore the $M$ mode "cubing method" may not be valid.

\section{Echo Doppler methods}

The product of flow velocities recorded by pulsed or continuous wave Doppler ultrasound with cross sectional area measured from cross sectional images is an accepted way of calculating stroke volume and cardiac output. There are several technical variations and the inherent assumptions are well reviewed by Goldberg $e t$ al. ${ }^{9}$ Since 1987 the technique has been used for a series of studies of the normal maternal heart during pregnancy in our department. ${ }^{10}$ In our early validatory studies cardiac output was calculated from the pulmonary, mitral, and aortic valves, which allowed us a "within patient" control for the values recorded. We initially validated these results from the aortic, pulmonary, and mitral valves against simultaneous direct Fick measurements. The correlation was good and the limits of agreement were $\pm 0.8 \mathrm{l} / \mathrm{min} .{ }^{10} \mathrm{We}$ also studied within patient, intraobserver, and chronological coefficients of variation for the measurements. These were all equal to or less than $5 \%$ for each of the three sites studied. The technique was then applied systematically to several -serial studies of cardiac output in pregnant subjects. The results form the basis for the summary of haemodynamic changes that follows.

Haemodynamic changes during normal singleton pregnancy

Our initial longitudinal studies of cardiac output started before conception and continued through to the postnatal period. Because of the effect on cardiac output of the supine position, which leads to caval occlusion by the gravid uterus, ${ }^{11}$ throughout all the studies measurements were performed in the left half lateral position.

Cardiac output had increased by five weeks after the last menstrual period and the increase continued to 24 weeks when it was $45 \%$ above the non-pregnant level (fig 1). Thereafter no further significant change was found. Both heart rate and stroke volume contributed to this increase: the heart rate increase was seen by five weeks' gestation and continued till 32 weeks. The stroke volume increase occurred a little later at eight weeks and reached its maximum at 
Figure 1 Increase in cardiac output from the non-pregnancy state throughout pregnancy. $P-P$, pre-pregnancy; $P N$, postnatal.
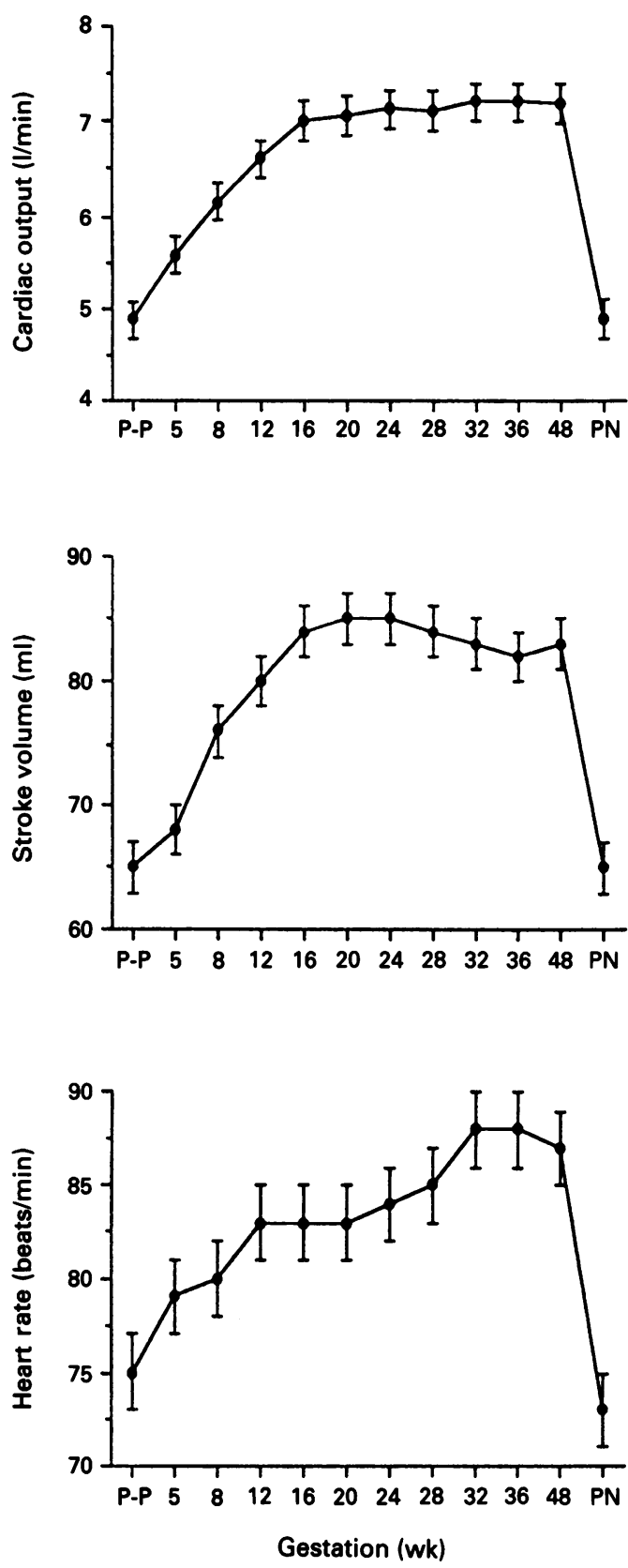

about 20 weeks. There was a slight fall in stroke volume after 38 weeks but this was not significant. However, other workers reported a fall in stroke volume in the last month of pregnancy. ${ }^{12}$

These results suggest that cardiac output increases earlier than previously reported. Cardiac output reached a maximum at the end of the second trimester. The maximum values and the time at which they were achieved resembled other reports. ${ }^{812-15}$ Similarly the reduction in cardiac output after pregnancy is similar to the $43 \%$ reduction reported by Clark et al in $1989 . .^{16}$

\section{Haemodynamic changes during twin pregnancy}

We had the opportunity to carry out a serial study of haemodynamic function during twin pregnancy. ${ }^{17}$ Cardiac output was on average
$15 \%$ higher throughout pregnancy compared with singleton pregnancy. This increase was due to a relatively greater increase in heart rate and was associated with a significantly larger increase in left atrial diameter representing increased volume overload.

\section{Haemodynamic changes in labour}

Previous studies of the haemodynamic changes during labour had suggested that there were dramatic changes in cardiac output. Our study of normal labour in women without epidural anaesthesia reported an increase in basal cardiac output (between contractions) of $12 \%$ that was mainly the result of an increase in stroke volume and occurred during the first stage of labour (fig 2). Cardiac output during contraction increased progressively as labour advanced till, at full dilatation of the cervix, cardiac output had increased by a mean value of $34 \%$. Early on in the first stage of labour this increase in cardiac output during contractions seemed to be caused by an increase in stroke volume but later on heart rate also increased.

\section{Haemodynamic changes during the puer- perium}

A dramatic increase in stroke volume was reported immediately after delivery, possibly caused by the relief of caval occlusion. ${ }^{18}$ Cardiac output according to previous studies returned to non-pregnant values at six weeks. ${ }^{81318}$ In our studies both cardiac output and heart rate had returned to the pre-pregnant values by two weeks after delivery (fig 3). Stroke volume had also decreased by two weeks although there was a further small reduction up to six months after delivery. If this process was looked at even more closely it became obvious that for the first two days after delivery stroke volume remained at the high values seen during pregnancy and thereafter fell dramatically. Heart rate remained raised during the first 24 hours after delivery and fell away dramatically over the next 10 days. Cardiac output remained up for 24 hours and then fell progressively until the tenth day.

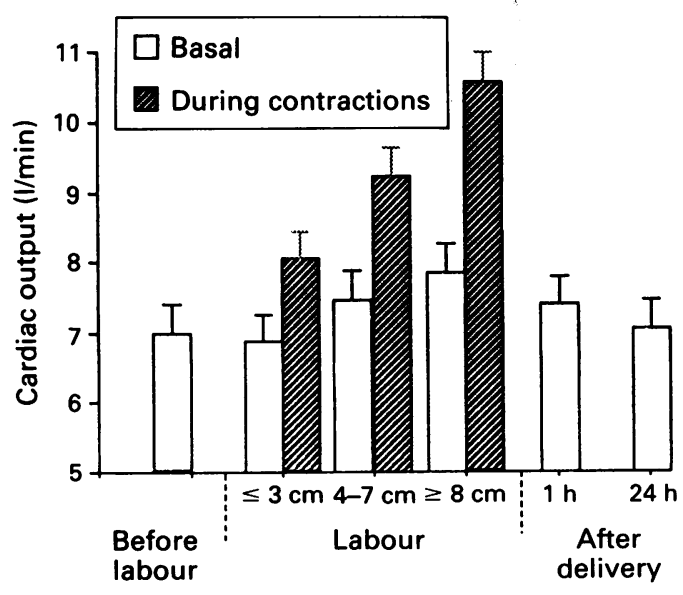

Figure 2 Changes in cardiac output and stroke volume during normal labour. 
Figure 3 Changes in heart rate and cardiac output after normal delivery
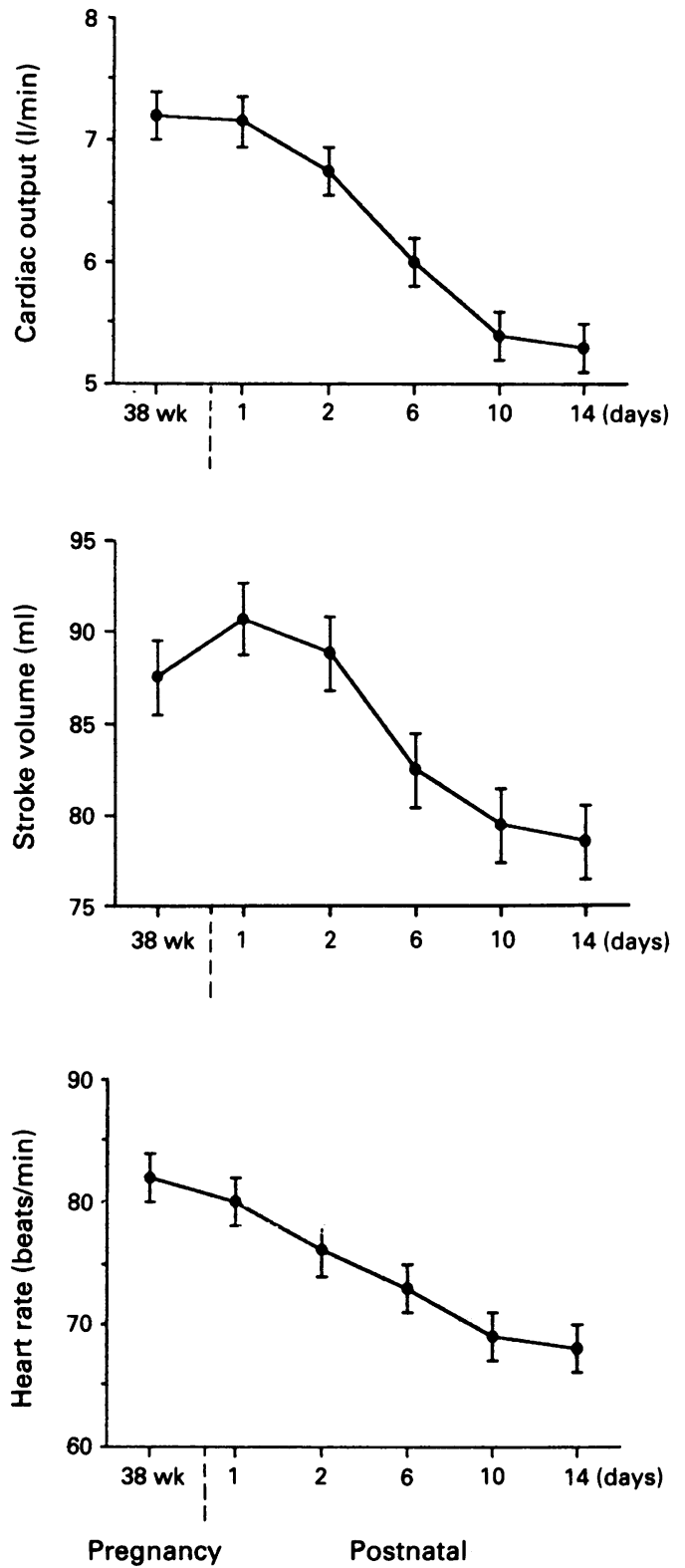

Structural cardiac changes secondary to pregnancy

During our serial study the cross sectional areas of the aortic, pulmonary, and mitral valves were measured throughout pregnancy. ${ }^{10}$ All three increased by between $12 \%$ and $14 \%$, the changes being evident by 12 weeks' gestation and continuing throughout pregnancy.

Haites et al described the aortic minute distance (velocity integral $\times$ heart rate) ${ }^{19}$ This index reflected changes in cardiac output in the non-pregnant state and had been suggested for use in pregnancy ${ }^{20}$ The increase in cross sectional area described above means that the use of stroke distance or minute distance as analogues of stroke volume or cardiac output in pregnancy will lead to an underestimate of the change shown by the full echo Doppler technique. Pregnancy produces an increase in left ventricular wall thickness and mass ${ }^{82} 22$ that is demonstrable during the second trimester and most marked at the end of pregnancy. In contrast to the functional changes, structural adaptation takes much longer (up to 12 weeks) regress after delivery. The analogy with the exercise effect in the normal heart is interesting.

Factors influencing the changes in cardiac output and function during pregnancy.

Venous return is bound to be increased during pregnancy probably because of the $40 \%$ increase in blood volume ${ }^{23}$ Serial measurement of left atrial and left ventricular end diastolic dimensions by $M$ mode support this. ${ }^{81424}$ The cavity dimension seemed to increase primarily during the second trimester at the time when plasma volume has been shown to rise. Despite this increase in volume the pressures within the lung vessels and the central venous pressures did not increase during pregnancy ${ }^{16}$ possibly because of ventricular dilatation and reduction in vascular resistance in both the systemic and pulmonary beds.

There is little doubt that blood pressure falls serially during pregnancy reaching its lowest level at about 20 weeks' gestation. ${ }^{24} 25$ From then until term there is a progressive increase in blood pressure both systolic and diastolic. Overall the fall in diastolic pressure is greater than the systolic change and the total systemic resistance falls considerably during pregnancy. In our own study systemic vascular resistance had fallen by $34 \%$ at 20 weeks' gestation. ${ }^{24}$ Thereafter there was a small increase to term. Overall these results suggest that cardiac afterload is significantly reduced during pregnancy possibly associated with a fall in aortic compliance and in viscosity.

Myocardial contractility is the third component of cardiac function. It is extremely difficult to assess in vivo although several studies of $M$ mode indices have shown that ejection fraction and mean rate of circumferential fibre shortening increase during pregnancy. ${ }^{212426}$ This increase occurs during the first two trimesters and seems to fall away during the third. The major problem lies in the interpretation of these findings because all these indices are affected by changes in heart rate, preload, and afterload. The only group to have studied intrinsic left ventricular contractility by the left ventricular work index is Clark et al.$^{16}$ In normal pregnant subjects they found a statistically insignificant increase at 36-38 weeks' gestation compared with postnatal control values.

\section{Summary}

The first haemodynamic change during pregnancy seems to be a rise in heart rate. Starting between two and five weeks this continues well into the third trimester. Stroke volume increases slightly later than the heart rate and continues throughout the second trimester after an augmentation of venous return and a fall of systemic vascular resistance and afterload. Myocardial contractility is probably slightly increased. During the third trimester there is relatively little change in these cardiac indices. After delivery there is a very early and 
dramatic reduction in volume loading followed by a return towards normal cardiac output. Structural changes within the heart reflect the volume loading of pregnancy and include dilatation of the valve ring and increase in myocardial thickness. Post partum resolution of the ventricular hypertrophy seems to take longer than the rest of the post partum changes. The resemblance to the cardiovascular changes associated with training and exercise are fascinating and worthy of further study.

1 Linhard J. Uber das Minutenvolum des Herzens bei Ruhe und bei Muskelarbeit. Pflugers Arch 1915;161:223-53.

2 Kubicek WG, Karnegis JN, Patterson RP, Witsoe DA Mattson RH. Development and evaluation of an impedance cardiac output system. Aerospace Med 1966;37:1208-12.

3 Secher NJ, Arnsbo P, Heslet Anderson L, Thompson A. Measurement of cardiac stroke volume in various body positions in pregnancy and during caesarian section: a positions in pregnancy and during caesarian section: a
comparison between thermodilution and impedance carcomparison between thermodilution and imped.

4 de Swiet M, Talbert DG. The measurement of cardiac Swiet $M$, Talbert DG. The measurement of cardiac
output by electrical impedance plethysmography. Are the output by electrical impedance plethysmography. Are the
assumptions valid? Br J Obstet Gynaecol 1986;93:721-6.

5 Pombo JF, Troy BL, Russell RO. Left ventricular volumes and ejection fraction by echocardiography. Circulation 1971;43:480-90

6 Fortuin NJ, Wood WP, Sherman ME, Craige E. Determination of left ventricular volume by ultrasound. Circulation 1971;44:575-84.

7 Ihrman K. A clinical and physiological study of pregnancy in a material from Northern Sweden. VII. The heart volume during and after pregnancy. Acta Soc Med Upsalien 1960;65:326-34.

8 Katz R, Karliner JS, Resnik R. Effects of a natural volume overload state (pregnancy) on left ventricular performance in normal human subjects. Circulation 1978;58:434-41.

9 Goldberg SJ, Allen HD, Marx GR, Donnerstein RL, eds. Flow computation. Doppler echocardiography. PhiladelFlow computation. Doppler echocardio

10 Robson SC, Dunlop W, Moore M, Hunter S. Combined Doppler and echocardiographic measurement of cardiac output: theory and application in pregnancy. Br J Obstet Gynaecol 1987;94:1014-27.
11 Lees MM, Scott DB, Kerr MG, Taylor SH. The circulatory effects of recumbent postural change in late pregnancy. Clin Sci 1967;32:453-65.

12 Ueland K, Novy MJ, Peterson EN, Metcalf J. Materna cardiovascular dynamics. IV. The influence of gestationa age on the maternal cardiovascular response to posture and exercise. Am J Obstet Gynecol 1969a;104:856-64.

13 Atkins AJF, Watt JM, Milan P, Davies P, Selwyn Crawford $\mathrm{J}$. A longitudinal study of cardiovascular dynamic changes throughout pregnancy. Eur J Obstet Gynecol Reprod Biol 1981;12:215-24.

14 Hirata F, Nishida N, Kanamura S, et al. Non-invasive estimates of hemodynamics in normal pregnancy. J Carestimates of hemodynam

15 Mashini IS, Albazzaz SJ, Fadel HE, et al. Serial noninvasive evaluation of cardiovascular hemodynamics during pregnancy. Am J Obstet Gynecol 1987;156:1208-13.

16 Clark SL, Cotton DB, Lee W, et al. Central hemodynamic assessment of normal term pregnancy. Am J Obstet Gynecol 1989;161:1439-42.

17 Robson SC, Hunter S, Boys RJ, Dunlop W. Hemodynamic changes during twin pregnancy. A Doppler and M-mode echocardiographic study. Am J Obstet Gynecol 1989;161:1273-8.

18 Ueland K, Hansen JM. Maternal cardiovascular dynamics. III. Labor and delivery under local and caudal analgesia. Am J Obstet Gynecol 1969;103:8-18

19 Haites NE, McLennan F, Mowat DHR, Rawles JM Assessment of cardiac output by the Doppler ultrasound Assessment of cardiac output by the Dopp

20 McLennan FM, Haites NE, Rawles JM. Stroke and minute distance in pregnancy: a longitudinal study using Dopple ultrasound. Br J Obstet Gynaecol 1987;94:499-506.

21 Laird-Meeter K, Van De Ley G, Bom TH, Wladimiroff JW Cardiocirculatory adjustments during pregnancy: An echocardiographic study. Clin Cardiol 1979;2:328-32.

22 Robson SC, Boys RJ, Hunter S, Dunlop W. Materna hemodynamics after normal delivery and delivery complicated by postpartum hemorrhage. Obstet Gynecol 1989;74:234-9.

23 Hytten FE. Blood volume changes in normal pregnancy. In: Letsky EA, ed. Clinics in haematology. vol 14 . London: WB Saunders, 1985:601-12.

24 Robson SC, Hunter S, Boys RJ, Dunlop W. Serial study of factors influencing changes in cardiac output during factors influencing changes in cardiac output during

25 MacGillivray I, Rose GA, Rowe B. Blood pressure survey in pregnancy. Clin Sci 1969;37:395-407.

26 Castillon G. Weissenburger J, Rouffet M, Castillon V, Barrat J. Etude échocardiographique des modifications hémodynamiques de la grossesse. J Gynecol Obstet Biol Reprod 1984;13:499-505. 